\title{
PUNÇÄO ASPIRATIVA NOS TUMORES DAS GLÂNDULAS SALIVARES: ESPECIFICIDADE E SENSIBILIDADE
}

\author{
Antonio José Gonçalves*, Marcelo Benedito Menezes, Norberto Kodi Kavabata, Antonio Augusto tupinambá Bertelli, Ricardo Antenor \\ de Souza e Souza, Daniel Joelsons \\ Trabalho realizado na Disciplina de Cirurgia de Cabeça e Pescoço do Departamento de Cirurgia da Faculdade de Ciências Médicas da Santa Casa de São Paulo
}

*Correspondência

Disciplina de Cirurgia de

Cabeça e Pescoço

Departamento de Cirurgia

Faculdade de Ciências Médicas

da Santa Casa de São Paulo,

R. Dr. Cesário Mota Jr., 61

Vila Buarque - São Paulo - SP

Brasil. Cep: 01221-020

cabpesc@santacasasp.org.br

\section{RESUMO}

As neoplasias das glândulas salivares são afecções que constituem aproximadamente 3\% de todos os tumores da cabeça e pescoço. $\mathrm{O}$ tratamento cirúrgico adequado depende do correto conhecimento do tipo histológico, principalmente quando se trata de lesões malignas. Dessa maneira, a correta determinação do diagnóstico histológico pré-operatório auxilia em muito o planejamento terapêutico, porém, geralmente, isto é difícil de se estabelecer baseado apenas nos achados da história, exame clínico e de imagem.

Oвjetivos. $O$ presente estudo avalia comparativamente os resultados de exames citológicos obtidos através de punção aspirativa com agulha fina (PAAF) de tumores de glândulas salivares, realizados no período pré-operatório, com os dados histológicos de espécimes cirúrgicos, analisando a sensibilidade, especificidade e acurácia do método quanto ao diagnóstico de malignidade e benignidade.

Métodos. Estudo retrospectivo de 73 prontuários de pacientes portadores de neoplasia de glândulas salivares, todos submetidos a tratamento cirúrgico.

Resultados. Obtivemos 87,9\% de sensibilidade e 85,7\% de especificidade para diagnóstico de tumores benignos; sensibilidade de $42,9 \%$ e especificidade de $98,3 \%$ para diagnóstico de tumores malignos. O valor global da acurácia foi de $87,7 \%$, o valor preditivo positivo de $85,7 \%$ e o negativo de $87,8 \%$ para diagnóstico de malignidade com a PAAF.

Conclusão. Concluiu-se que a PAAF como método diagnóstico auxiliar, pode ser útil na avaliação pré-operatória, especialmente se o diagnóstico for de malignidade, contribuindo assim para um melhor planejamento cirúrgico do doente.

Unitermos: Sensibilidade. Especificidade. Punção aspirativa por agulha fina. Glândula salivar. Neoplasia.

\section{INTRODUÇÃO}

As neoplasias das glândulas salivares são afecções incomuns e constituem aproximadamente 3\% de todos os tumores da cabeça e pescoço $0^{2-3}$ e caracterizados por uma grande heterogeneidade do ponto de vista histológico, comportamento biológico e evolução clínica-6. $\mathrm{O}$ tratamento cirúrgico adequado depende, muitas vezes, do tipo histológico envolvido, principalmente quando se trata de lesões malignas. $^{5}$ Dessa maneira, o conhecimento do diagnóstico histológico pré-operatório auxilia em muito o planejamento terapêutico ${ }^{7}$, o esclarecimento e preparo adequado do paciente quanto à extensão da cirurgia!, principalmente nos tumores malignos em estádio precoce ${ }^{7}$, - que geralmente é difícil de se estabelecer baseado apenas nos achados da história, exame clínico e de imagem ${ }^{8-9}$.

A punção biópsia aspirativa por agulha fina (PAAF) utilizada pela primeira vez na investigação de lesões de glândulas salivares em 1927 por Dudgeon \& Patrick ${ }^{10}$ e em 1930 por Martin \& Ellis" tornou-se método corriqueiro somente na década de $1970^{12}$ e, desde então, diversos estudos têm avaliado este método citológico nas lesões de glândulas salivares, com opiniões diferentes quanto ao valor da sua utilização na prática clínica'2-13. O presente estudo foi realizado com objetivo de avaliar comparativamente os resultados obtidos em PAAF de tumores de glândulas salivares realizados no período pré-operatório com os dos respectivos exames histológicos dos espécimes cirúrgicos analisando a sensibilidade, especificidade e acurácia do método quanto ao diagnóstico de malignidade e benignidade, além do diagnóstico sugerido pela PAAF em relação ao tipo histológico.

\section{Métodos}

Trata-se de estudo retrospectivo com revisão de 73 prontuários de pacientes portadores de neoplasia de glândulas salivares, submetidos a tratamento cirúrgico pela Disciplina de Cirurgia de Cabeça e Pescoço do Departamento de Cirurgía da Faculdade de Ciências Médicas da Santa Casa de São Paulo no período de fevereiro de 1997 a dezembro de 2001 . Destes, 33 (45,2\%) eram homens e 40 (54,8\%) mulheres; a média de idade foi de 53,5 anos (variando de 9 a 76 anos). A maioria dos casos localizava-se na parótida (78\%) e glândula submandibular (19,1\%). Os outros tumores eram de glândula salivar menor e nenhum caso neste estudo localizava-se em glândula sub-lingual.

As PAAF foram realizadas por médico citopatologista após assepsia adequada da pele, seguida de punção com agulha 
GONÇALVES AJ ET AL.

25X6mm. e seringas de 10 millitros, conectadas a dispositivo para obtenção de pressão negativa. Um mínimo de duas punções foi realizado em cada caso. O material obtido pelas punções foi expelido sobre quatro ou cinco lâminas de microscopia e submetido à técnica de esfregaço, sendo imediatamente fixadas em álcool absoluto ou a seco e posteriormente coradas pelos métodos de hematoxilinaeosina e MGG (May Greenwald Giemsa) respectivamente. Todas as lâminas foram analisadas pelo mesmo citopatologista tentando sempre estabelecer o diagnóstico do tipo tumoral envolvido.

Os espécimes cirúrgicos fixados em formalina a 10\% foram encaminhados ao laboratório de anatomia patológica, onde foi realizado o exame macroscópico. Identificando-se o tumor, este foi mensurado e feito cortes representativos de 5 milímetros de espessura. Estes foram colocados em cassetes e processados em autotécnico por 9 horas, passando por concentrações progressivas de álcool e xilol. Posteriormente, o material foi incluído em parafina e realizado corte histológico de quatro micras. Após desparafinização em estufa, as lâminas foram submetidas à coloração de rotina pelo método de hematoxilina-eosina. Todas as lâminas foram analisadas pelo mesmo anátomo-patologista tentando sempre estabelecer o diagnóstico do tipo tumoral envolvido.

Os resultados dos exames citológicos e anátomo-patológicos foram analisados comparando-se os diagnósticos sugeridos pela PAAF e os resultados do exame histopatológico, além de identificar os resultados falsos-positivos e falsos-negativos, e calcular a sensibilidade, especificidade, acurácia e valor preditivo positivo e negativo da PAAF.

A sensibilidade foi calculada baseada na razão entre os resultados verdadeiramente positivos pelo total de tumores confirmadamente malignos. A especificidade foi calculada através da razão entre os resultados verdadeiramente negativos pelo total de tumores confirmadamente benignos. A acurácia foi calculada através da razão entre o número total de verdadeiros positivos e verdadeiros negativos pelo número total de tumores analisados. $O$ valor preditivo positivo foi calculado através da razão entre os resultados verdadeiramente positivos pelo número total de falsos-positivos mais verdadeiros positivos, e o valor preditivo negativo através da razão entre os resultados verdadeiramente negativos pelo número total de falsos-positivos mais vardadeiros-negativos.

\section{Resultados}

Dos exames de PAAF, 66 (90,4\%) foram considerados benignos e sete $(9,6 \%)$ malignos, porém, desse montante, oito (I2\%) eram casos falsos-negativos e um caso (14,3\%) era falso-positivo (o anatomopatológico definitivo demonstrou tratar-se de adenoma pleomorfo).

$\mathrm{Na}$ Tabela I temos a correlação entre os resultados obtidos pela PAAF e os anatomopatológicos dos espécimes cirúrgicos dos tumores benignos e malignos.

Como visto na tabela acima, dos 42 casos de adenoma pleomorfo, 34 (80,9\%) foram corretamente diagnosticados pela PAAF. No tumor de Warthin tivemos um índice de acerto menor de $72,7 \%(8 / 1 \mathrm{I})$. Nos demais tipos, a PAAF não conseguiu

\begin{tabular}{|c|c|c|}
\hline \multicolumn{3}{|c|}{$\begin{array}{l}\text { Tabela I - Correlação entre os resultados da PAAF e dos exames } \\
\text { histopatológicos nos tumores de glândulas salivares }\end{array}$} \\
\hline AP definitivo & PAAF & no. casos \\
\hline $\begin{array}{l}\text { Adenoma pleomorfo } \\
\text { ( } 42 \text { casos) }\end{array}$ & $\begin{array}{l}\text { Adenoma pleomorfo } \\
\text { Neoplasia de cél. Epiteliais-mioepiteliais } \\
\text { Neoplasia de cel. Basais } \\
\text { Conteúdo cístico } \\
\text { Ca em adenoma pleomorfo }\end{array}$ & $\begin{array}{l}34 \\
03 \\
03 \\
01 \\
01\end{array}$ \\
\hline $\begin{array}{l}\text { Tumor de Warthin } \\
\text { (I I casos) }\end{array}$ & $\begin{array}{l}\text { Tumor de Warthin } \\
\text { Lesão linfoepitelial } \\
\text { Cisto branquial }\end{array}$ & $\begin{array}{l}08 \\
02 \\
01\end{array}$ \\
\hline $\begin{array}{l}\text { Lesão linfoepitelial } \\
\text { (3 casos) }\end{array}$ & $\begin{array}{l}\text { Lesão linfoepitelial } \\
\text { Conteúdo cístico }\end{array}$ & $\begin{array}{l}01 \\
02\end{array}$ \\
\hline Adenoma de cel. basais & Adenoma pleomorfo & 02 \\
\hline Mioepitelioma & Conteúdo cístico & 01 \\
\hline $\begin{array}{l}\text { Camucoepidermóide } \\
\text { (3 casos) }\end{array}$ & $\begin{array}{l}\text { Ca mucoepidermóide } \\
\text { Neoplasia de cel. basais } \\
\text { Neoplasia de cel. Epiteliais-mioepiteliais }\end{array}$ & $\begin{array}{l}01 \\
01 \\
01\end{array}$ \\
\hline $\begin{array}{l}\text { Caadenóide cístico } \\
\text { (3 casos) }\end{array}$ & $\begin{array}{l}\text { Carcinoma adenóide cístico } \\
\text { Neoplasia de cél. basais }\end{array}$ & $\begin{array}{l}02 \\
01\end{array}$ \\
\hline $\begin{array}{l}\text { Adenocarcinoma } \\
\text { (3 casos) }\end{array}$ & $\begin{array}{l}\text { Adenocarcinoma } \\
\text { Carcinoma } \\
\text { Neoplasia de cel. basais }\end{array}$ & $\begin{array}{l}01 \\
01 \\
01\end{array}$ \\
\hline Carcinoma de cel. Acinares & Neoplasia de cel. acinares & 02 \\
\hline Carcinomamioepitelial & Adenoma pleomorfo & 01 \\
\hline Ca emadenoma pleomorfo & Carcinoma em adenoma pleomorfo & 01 \\
\hline Linfoma & Lesão linfoepitelial & 01 \\
\hline TOTAL & & 73 \\
\hline
\end{tabular}

Fonte: SAME da F.C.M.S.C.S.P. - fevereiro del997 a dezembro de 2001

fornecer o correto diagnóstico da lesão, com exceção de um caso de lesão linfoepitelial. Porém o índice total de acento foi de 86,5\%.

Continuando a análise da Tabela I, observamos um índice de acerto somente do diagnóstico sugerido pela PAAF tanto nos tumores benignos como nos malignos, contudo, no que se refere à detecção de malignidade, houve acerto em 85,7\% dos casos (6) 7). No caso falso-positivo, a análise citológica mostrou características de adenoma pleomorfo associado a indícios de metaplasia escamosa, o que levou a suspeita de que tratava-se de carcinoma em adenoma pleomorfo.

Na Tabela 2 comparamos os resultados dos diagnósticos sugeridos pela PAAF com os resultados dos exames histopatológicos definitivos quanto à benignidade e malignidade dos casos. Observamos, então, que nos diagnósticos de adenoma pleomorfo, tumor de Warthin e lesões císticas à PAAF, 97,9\% (47/48) dos casos tratavamse realmente de afecções benignas. Nos casos em que o diagnóstico em que da PAAF foi carcinoma mucoepidermóide, carcinoma denóide-cístico e adenocarcinoma, apesar de poucos casos, todos mostraram-se verdadeiros, denotando uma ótima especificidade do método, apesar da baixa sensibilidade (a cada três casos destes tipos histológicos, apenas um foi identificado pela punção). 


\begin{tabular}{|c|c|c|c|}
\hline \multirow[b]{2}{*}{ Laudo PAAF } & \multicolumn{2}{|c|}{ Examehispatológico } & \multirow[b]{2}{*}{ Tota } \\
\hline & benigno & $\underline{\text { maligno }}$ & \\
\hline Adenoma pleomorfo & $36(97,3 \%)$ & $01(2,7 \%)$ & 37 \\
\hline Tumor de Warthin & $08(100 \%)$ & - & 08 \\
\hline Conteúdo cístico & $04(100 \%)$ & - & 04 \\
\hline Neoplasia de cél. epit.-mioepiteliais & $03(75 \%)$ & $01(25 \%)$ & 04 \\
\hline Neoplasia de cel. basais & $03(50 \%)$ & $03(50 \%)$ & 06 \\
\hline Lesão linfofoepitelial & $03(75 \%)$ & $01(25 \%)$ & 04 \\
\hline Neoplasia de cel.acinares & 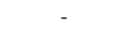 & $02(100 \%)$ & 27 \\
\hline Carcinoma mucoepidermóide & - & $01(100 \%)$ & ol \\
\hline Carcinoma adenóide cístico & - & $02(100 \%)$ & 02 \\
\hline Adenocarcinoma & - & $01(100 \%)$ & 01 \\
\hline
\end{tabular}

Fonte: SAME da F.C.M.S.C.S.P. - fevereiro del 1997 a dezembro de 2001

\begin{tabular}{|c|c|c|c|}
\hline \multirow[b]{2}{*}{ Resultadohistopatológico } & \multicolumn{2}{|c|}{ PAAF } & \multirow[b]{2}{*}{ Tota } \\
\hline & Benigno & Maligno & \\
\hline Benigno & 58 & 01 & 59 \\
\hline Maligno & 8 & 06 & 14 \\
\hline Total & 66 & 07 & 73 \\
\hline
\end{tabular}

Fonte: SAME da F.C.M.S.C.S.P. - fevereiro del997 a dezembro de 2001

Considerando-se a malignidade nos exames de PAAF, tivemos 58 casos verdadeiramente negativos, seis casos verdadeiramente positivos, um caso falso-positivo e oito casos falsos-negativos (Tabela 3). Baseados nestes dados, calculamos os valores de sensibilidade $(87,9 \%)$ e especificidade $(85,7 \%)$ para diagnóstico de tumores benignos; sensibilidade (42,9\%) e especificidade $(98,3 \%)$ para diagnóstico de tumores malignos, valor global da acurácia (87,7\%), valor preditivo positivo $(85,7 \%)$ e negativo $(87,8 \%)$ para diagnóstico de malignidade da PAAF. Apesar do valor preditivo negativo ser de $87,8 \%$, a PAAF mostrou, nesta casuística, uma baixa sensibilidade $(42,9 \%)$ porque tivemos $57,1 \%(8 / 14)$ de falsos-negativos. A especificidade (98,3\%), por outro lado, foi alta pois tivemos apenas I,7\% (I/59) de falsos-positivos.

\section{Discussão}

tratamento dos tumores malignos e benignos de glândulas salivares difere de modo significativo. No grupo dos tumores malignos temos necessidade de cirurgias mais radicais, eventualmente associadas ao tratamento concomitante das cadeias de disseminação linfática, diante de uma preocupação significativamente maior com a possibilidade de recidivas ${ }^{14-15}$, além da maior gravidade da doença. É óbvia a importância do tratamento precoce nas neoplasias malignas, no qual as margens cirúrgicas adequadas são fundamentais ${ }^{16-17}$, a fim de melhorar as taxas de controle local, uma vez que a sua falha está fortemente relacionada ao desenvolvimento de metástases distantes e a uma pior sobrevivência dos pacientes ${ }^{4}$ 7,15,18-20. A PAAF parece ter papel importante no que diz respeito à possibilidade de um diagnóstico mais preciso e, assim, a possibilidade de um melhor planejamento terapêutico pré-operatório e a possibilidade de discussão da maior ou menor extensão da cirurgia com os pacientes 1, 12,21

A suspeita diagnóstica de malignidade em tumores de glândulas salivares é relativamente fácil nos casos mais avançados, conseqüentemente os de pior prognóstico, pois já são observados comprometimento nervoso, dor importante, ulcerações na pele, infiltração de tecidos adjacentes, metástases à distância, entre outros. Contudo, em pequenas tumorações, esse diagnóstico nem sempre é possível de ser feito baseado apenas na história clínica, achados de exame físico e até mesmo de exames radiológicos ${ }^{8,22}$.

Apesar da possibilidade de utilizarmos o exame histopatológico de congelação durante 0 procedimento cirúrgico para confirmação diagnóstica dos tumores de glândulas salivares, temos obtido resultados semelhantes e, às vezes, piores do que a PAAF, concordantes com os achados de Heller et al..$^{23}$ que relatam sensibilidade de $69 \%$, especificidade de $96 \%$ e acurácia de $51 \%$ para detecção de malignidade em exames de congelação para tumores de glândulas salivares.

Assim sendo, a PAAF têm sido utilizada na avaliação diagnóstica de tumorações de glândulas salivares, mostrando ser um método seguro e com raras complicações 1,12,21,24. Em nossa casuística não tivemos nenhum tipo de complicação significativa.

Nem todos, contudo, concordam quanto a utilidade clínica da PAAF, pela sua incapacidade de avaliar a extensão tumoral, a relação do tumor com o nervo facial entre outros ${ }^{9}$, porém, acreditamos que a sua grande importância está na possibilidade de diagnosticar as lesões malignas em tumores de estádio precoce, uma vez que diversos autores têm demonstrado resultados satisfatórios, com sensibilidade para diagnóstico de tumores malignos variando entre $58 \%$ a $96 \%$ e especificidade de $71 \%$ a $95 \% \%^{12,21,25-26}$. Apesar de Batsakis et al. ${ }^{9}$ terem observado alterações teciduais em neoplasias de glândulas salivares decorrentes da PAAF que podem dificultar o diagnóstico histopatológico, em nossa experiência não houve dificuldades ou impedimentos diagnósticos em conseqüência da PAAF.

O rigor com que o citopatologista analisou os casos, principalmente com respeito à malignidade, pode favorecer um menor índice de falsos-positivos, uma vez que o diagnóstico de tumor maligno só foi considerado quando haviam critérios que o mesmo julgava suficientes para tal, porém, tal rigor não conseguiu detectar todas as neoplasias malignas, denotando a importância da experiência do examinador no estudo citopatológico de tumores de glândulas salivares.

Em nosso estudo, tivemos uma sensibilidade menor do que a relatada na literatura com valor de $42,9 \%$, pelo elevado número de falsos-negativos, problema esse também observado por Geisinger \& Weidner ${ }^{27}$. A especificidade encontrada de $98,3 \%$ está, por outro lado, em conformidade com outros estudos, assim como a acurácia de $87,7 \%$. 
Gonçalves Al et Al.

Importante comentar a alta sensibilidade e especificidade do método em alguns diagnósticos específicos, como nos casos de adenoma pleomorfo, tumor de Warthin e cisto glandular onde a margem de erro foi mínima se levada em conta apenas benignidade e malignidade. Apenas um caso dos 35 com resultado citológico de adenoma pleomorfo tratava-se de lesão maligna (carcinoma mioepitelial).

Dessa maneira, a PAAF auxiliou na estratégia cirúrgica préoperatória principalmente nos diagnósticos de malignidade, assim concordamos com Shara et al. ${ }^{1,28}$ que sustentam a opinião de que somente os achados positivos para malignidade devem efetivamente ser considerados. Observamos que o método, numa maior casuística, pode mostrar-se bastante sensível para o diagnóstico dos tumores mais comuns, carcinoma muco-epidermóide e carcinoma adenóide-cístico, das glândulas salivares. Já os resultados negativos, especialmente aqueles com resultados diferentes de adenoma pleomorfo, tumor de Warthin e conteúdo cístico, devem ser criteriosamente analisados com base no quadro clínico do paciente.

\section{Conclusóes}

O presente estudo sugere que a PAAF, como método diagnóstico auxiliar, é útil na avaliação pré-operatória, especialmente quando o diagnóstico à PAAF é de malignidade. Por outro lado, os resultados negativos para malignidade devem ser criteriosamente analisados, pois a possibilidade de processo neoplásico maligno não é desprezível, principalmente quando tratar-se de diagnósticos sugeridos diferentes de adenoma pleomorfo, tumor de Warthin e lesões císticas.

\section{Conflito de interesse: não há}

\section{SUMMARY}

FINE NEEDLE ASPIRATION IN SALIVARY GLAND TUMORS: SPECIFICITY AND SENSITIVITY

Neoplasms of salivary glands represent almost $3 \%$ of all head and neck tumors. Proper surgical treatment depends upon accurate histological findings, especially in the case of malignant lesions. As such, knowledge of correct cytological findings prior to surgery is important for therapeutic planning. This is not easily established since it is usually based only on the patients' clinical history and imaging exams

OBJECTIVE. To evaluate results obtained from fine needle aspiration biopsy (FNAB), comparing them to the histological findings of the respective surgical specimens and analyzing the sensitivity, specificity and accuracy of this method in relation to the diagnosis of malignancy or benignancy.

METHODS. Retrospective study with the medical records of $73 e e$ patients' who had salivary gland neoplasms and were submitted to FNAB and surgical treatment.

RESULTS. Data disclosed that values of sensitivity were $87.9 \%$ and specificity $85.7 \%$ for diagnosis of benign tumors. For malignant tumors $42.5 \%$ of sensitivity and 98.3 of specificity, were observed. Overall values of accuracy were, respectively, $85.7 \%$ and $87.8 \%$ for positive and negative predictive values in the diagnosis of malignancy by FNAB.

CONCLUSIONS. It was shown that FNAB, as supplementary diagnostic method, can be useful for preoperative evaluation and surgical planning especially for malignant neoplasms. [Rev Assoc Med Bras 2007; 53(3): 267-7I]

KEY wORDS: Sensivity. Specificity. Accuracy. Fine needle aspiration biopsy. Salivary gland neoplasia.

\section{REFERÊNCIAS}

I. Shaha AR, Webber C, DiMaio T, Jaffe BM.- Needle aspiration in salivary gland lesions. Am.J.Surg. 1990;160:373-6.

2. Calearo C, Pastore A, Storchi OF, Polli G. Parotid gland carcinoma: Analysis of prognostic factors. Ann Otol Rhinol Laryngol. 1998: 107:969-73.

3. Eneroth CM. Incidence and prognosis of salivary gland tumors at different sites. A study of parotid, submandibular and palatal tumors in 2632 patients. Acta Otolaryngol. 1970;263:174-8.

4. Godballe C, Schultz JH, Krogdahl A, Møller-Grøntved ÅJ. Parotid carcinoma: Impact of clinical factors on prognosis in a histologically revised series. Laryngoscope. 2003;1|3:14||-7.

5. Therkildsen MH, Christensen M, Andersen LJ, Schiødt T, Hansen HS. Salivary gland carcinomas. Acta Oncol. 1998;37:701-13.

6. Pedersen D, Overgaard J, Søgaard $H$, Elbrønd $O$, Overgaard $M$. Malignant parotid tumors in 110 consecutive patients: Treatment results and prognosis. Laryngoscope. 1992;102:1064-9.

7. O'Brien CJ, Soong FSJ, Herrera GA, Urist MM, Maddox WA. Malignant salivary tumors: analysis of prognostic factors and survival. Head Neck Surg. 1986;9(2):82-92.

8. Zurrida S, Alasio L, Tradati N, Bartoli C, Chiesa F, Pilotti S. Fine-needle aspiration of parotid masses. Cancer. 1993;72:2306-11.

9. Owen EERTC, Banerjee AK, Prichard AJN, Hudson EA, Kark AE. Role of fine-needle aspiration citology and computed tomography in the diagnosis of parotid swellings. Br J Surg. 1989;76:1273-4.

10. Dudgeon LS, Patrick CV. A new method for the rapid microscopical diagnosis of tumours. $\mathrm{Br} \mid$ Surg. | 927:| 5:250-61.

I I. Martin HE, Ellis EB. Biopsy by needle puncture and aspiration. Ann Surg. 1930;92:169-81.

1 2. Zbären $\mathrm{P}$, Schär $\mathrm{C}$, Hotz MA, Loosli $\mathrm{H}$. Value of fine-needle aspiration cytology of parotid gland masses. Laryngoscope. 200 1; 1 1 1:1989-92.

13. Batsakis JG, Sneige N, El-Naggar AK. Pathology consultation. Fineneedle aspiration of salivary glands: Its utility and tissue effects. Ann Otol Rhinol Laryngol. 1992;101:|85-8.

14. Fitzpatrick PJ, Black KM. Salivary gland tumors. J Otolaryngol 1 985; | 4:296-300

15. Spiro RH. Salivary neoplasms: overview of a 35-year experience with 2,807 patients. Head Neck Surg. 1986;8:177-84.

16. Bradley PJ. Submandibular gland and minor salivary gland neoplasms. Curr Opin Otolaryngol Head Neck Surg. 2003;7:72-83.

17. Illes RW, Brian MB. A review of the tumors of the salivary gland. Surg Gynecol Obstet. 1986;163:399-404.

1 8. Tran L, Sadeghi A, Hanson D, Juillard G, Mackintosh R, Calcaterra TC, et al. Major salivary gland tumors: Treatment results and prognostic factors. Laryngoscope. 1986;96:1/39-44.

19. Byun YS, Fayos JV, Kim YH. Management of malignant salivary gland tumors. Laryngoscope. 1980;90:1052-60.

20. Spiro RH. Distant metastasis in adenoid cystic carcinoma of salivary origin. Am J Surg. 1997; 174:495-8. 
PUnÇÃO ASPIRATIVA NOS TUMORES DAS GLÂNDULAS SALIVARES: ESPECIFIIIDADE E SENSIBILIDADE

$2 \mathrm{I}$. Shintani S, Matsuura H, Hasegawa Y. Fine-needle aspiration of salivary gland tumors. Int J Oral Maxillofac Surg. 1997;26:284-6.

22. Yousem EK. Dashed hopes for MR imaging of the head and neck: The power of the needle. Radiology. 1992; 184:25-6.

23. Heller KS, Attie JN, Dubner S. Accuracy of frozen section in the evaluation of salivary tumors. Am .J Surg. 1993;166:424-7.

24. Tabbara SO, Frierson HF, Fechner RE. Diagnostic problems in tissues previously sampled by fine-needle aspiration. Am 」 Clin Pathol. 1991;96:76-80

25. Heller KS, Dubner S, Chess Q, Attie JN. Value of fine needle aspiration biopsy of salivary gland masses in clinical decision-making. Am J Surg. 1992; 1 64:667-70.
26. Frable MAS, Frable WJ. Fine-needle aspiration biopsy of salivary glands. Laryngoscope. 1991;101:245-9.

27. Geisinger KR, Weidner N. Aspiration cytology of salivary glands. Semin Diagn Pathol. 1986;3:219-26.

28. Shara AR, Weber CA, Marti JR. Fine needle aspiration in the diagnosis of cervical adenopathy. Am.J.Surg. 1986;152:420-4. 